\title{
Autonomous Energy Grids
}

\author{
Benjamin Kroposki \\ National Renewable \\ Energy Laboratory \\ Benjamin.kroposki \\ @nrel.gov
}

\author{
Emiliano Dall'Anese \\ National Renewable \\ Energy Laboratory \\ Emiliano.DallAnese \\ @nrel.gov
}

\author{
Andrey Bernstein \\ National Renewable \\ Energy Laboratory \\ Andrey.Bernstein \\ @)nrel.gov
}

\author{
Yingchen Zhang \\ National Renewable \\ Energy Laboratory \\ Yingchen.Zhang \\ @.nrel.gov
}

Bri-Mathias Hodge
National Renewable
Energy Laboratory
Bri.Mathias.Hodge
@nrel.gov

\begin{abstract}
Current frameworks to monitor, control, and optimize large-scale energy systems are becoming increasingly inadequate because of significantly high penetration levels of variable generation and distributed energy resources being integrated into electric power systems; the deluge of data from pervasive metering of energy grids; and a variety of new market mechanisms, including multilevel ancillary services. This paper outlines the concept of autonomous energy grids (AEGs). These systems are supported by a scalable, reconfigurable, and selforganizing information and control infrastructure, are extremely secure and resilient (self-healing), and can self-optimize in real time to ensure economic and reliable performance while systematically integrating energy in all forms. AEGs rely on cellular building blocks that can self-optimize when isolated from a larger grid and participate in optimal operation when interconnected to a larger grid. This paper describes the key concepts and research necessary in the broad domains of optimization theory, control theory, big data analytics, and complex system theory and modeling to realize the AEG vision.
\end{abstract}

\section{Introduction}

Energy systems generate, transport, convert, and consume energy. They encompass a wide range of domains, including electric power systems, thermal systems used for heating and cooling, and fuel systems such as natural gas or hydrogen networks. In addition, a large number of interrelated domains influence the operation of these energy systems, including communications, water, and transportation networks. Energy systems can function at a variety of physical scales, from a small individual consumer, to communities and cities, to larger regions that encompass transmission networks. Figure 1 shows these interrelationships at a variety of physical scales [1].
Current power systems deliver electricity primarily in one direction: flowing from large central plants to customer loads; however, increasing amounts of variable generation (wind and solar), distributed energy resources (DERs) (solar, fuel cells, microturbines, gensets), distributed energy storage (batteries, ice storage), and new loads (electric vehicles, lightemitting diode lighting) are being added to electric grids and causing bidirectional power flows and voltage fluctuations that impact optimal control and system operation. In addition, because of increased numbers and types of sensors, massive amounts of new data are being collected on energy grid conditions.

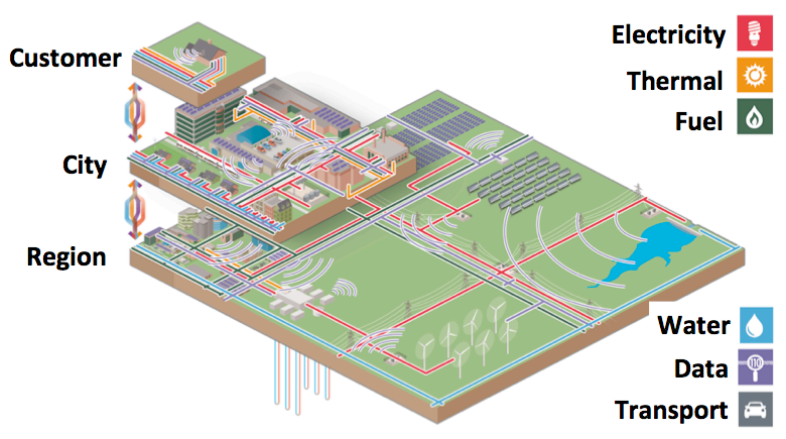

Figure 1. Energy systems integration occurs at a variety of physical scales.

The use of natural gas-fired generation has also increased significantly, both at the bulk level and locally through combined heat-and-power applications. Finally, new ideas such as islanded microgrids [2] are being used locally to disconnect parts of an energy system from a larger system for economic reasons and to improve customer reliability and resilience. The formation of microgrids can be seamless when there is no interruption to customer loads or they can isolate from the grid with a discontinuity of service.

Current grid control systems that operate at the bulksystem level typically control about 10,000 points even on the largest systems. As additional smart, controllable devices are integrated into the grid, the number of control points could easily reach hundreds 
of millions, significantly increasing the complexity of how to control and optimize the system. All these new technologies (Figure 2) are increasing the complexity of energy systems to a point at which existing techniques to monitor, control, and optimize them will be inadequate. For example, existing techniques might not offer decision-making capabilities that are consistent with the form and function of future largescale systems, which will be governed by faster dynamics, include heterogeneous energy assets that are controllable at different temporal resolutions, and accommodate a variety of deregulated energy markets [3]. This paper proposes the concept of autonomous energy grids (AEGs) and describes the research needed to fully exploit new techniques that enable their secure, resilient, and economic operation. Similar to autonomous vehicles - which do not require a physical driver and can make decisions on how to most effectively transport a person from one place to another-AEGs do not require physical operators, can be extremely secure and resilient (self-healing), and can self-optimize in real time to ensure economic and reliable performance while integrating energy in all forms. To achieve these goals, AEGs rely on scalable cellular blocks that can self-optimize when isolated from a larger grid and participate in optimal operation when interconnected to a larger grid. These scalable cells (Figure 2) can be areas of the grid that can run independently as microgrids or be parts of the grid that are segregated from a control perspective, but they do not have enough local generation to carry the full load of the cell. The AEG concept allows for the use of optimization and control across cells in cases when the cells can form independent microgrids and when they can control assets but not intentionally island.

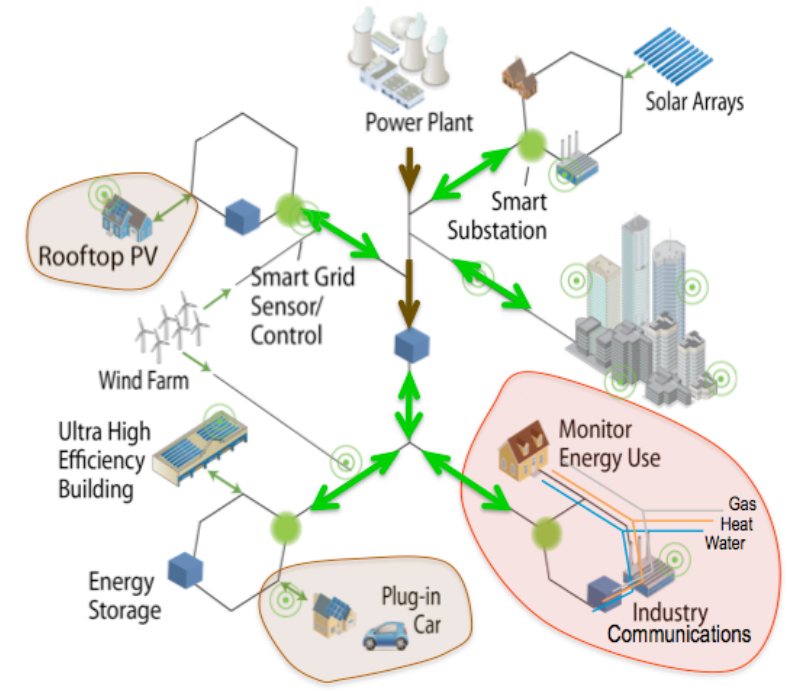

Figure 2. Energy systems incorporate a variety of energy sources and can form cells.
A critical factor that makes the operation of energy grids challenging is that underlying problems associated with control, optimization, and monitoring tasks need to be solved in real time and in a distributed fashion. Energy is constantly being produced and used, and this balance requires fast decision-making capabilities along with comprehensive situational awareness. Currently, energy systems of significant size make a large number of simplifications and rely on the underlying physics of the systems to operate properly. A further challenge is associated with the range of timescales that are important to the real-time operation of energy grids. In power systems, this can range from ultrafast protection schemes, through transient and dynamic stability in the seconds range, to power flow and unit commitment, which can be hours and days.

As more flexibility [4], [5] is added to energy systems, the complexity of controlling and optimizing these systems will increase to a point at which they will be too difficult to manage using conventional techniques. Future energy systems will need techniques that fully use big data analytics and advances in optimization, control, and complex system theories. As the grid evolves, there will also be challenges to operation caused by the ownership of various controllable assets. Because distributed generation, storage, and local loads might be under the ownership of customers instead of power system operators, how these devices participate in real-time operations will need to be further defined to ensure grid stability and reliability.

\section{Real-Time Optimization}

This section focuses on real-time optimization methods for AEGs. The term real time refers to an operational setting in which the power set points of the DERs within each cell are updated on a second or subsecond timescale to maximize the operational and economic objectives while coping with the variability of ambient conditions and noncontrollable energy assets [4] and achieving intercell coordination to ensure reliable system-wide operation. The main challenge in this context is related to the solution of pertinent optimization problems at a timescale that matches the dynamics of the prevailing ambient conditions, noncontrollable assets, and changes in the AEGs' configurations. In fact, solving optimization problems to convergence (i.e., in a batch setting) every second or every few seconds is currently impractical because of the following challenges:

(c1) Complexity and convergence rate. For large-scale grids, computational complexity might prevent the 
solution of optimization problems at the required timescales [3], [6]. When an optimization problem is solved in a distributed fashion (e.g., with cell-to-cell communications as well as intracellular message passing), multiple communication rounds are necessary to converge to (possibly optimal) solutions.

(c2) Model inaccuracy. Approximate linear models or convex relaxation methods might be leveraged to derive convex problems that facilitate the design of computationally affordable solutions; however, approximate/relaxed convex problems might involve only an approximate representation of a system's physics and constraints, and therefore the optimal solutions of the convex problem might not be feasible for the original problem.

(c3) Pervasive metering. Solving optimization problems such as relaxations/linearizations of the AC optimal power flow (OPF) requires pervasive metering to collect measurements of the noncontrollable loads at all locations in real time, which might be impractical.

Regarding (c1), note that the optimization tasks related to AEGs are markedly different from traditional settings wherein energy systems are optimized at the wholesale level using economic- and market-based objectives. The modus operandi in bulk systemswherein a few large-scale generators need to be dispatched and the noncontrollable net load varies slowly - is not compatible with the form and function of AEGs with massive integrations of DERs or when optimization models require accurate representations of the AC power flows as well as the controllability region of the DERs. In these cases, optimization problems are nonconvex, nondeterministic polynomialtime hard (NP-hard), and therefore they are infeasible to solve at the envisioned timescale [3], [6]. Nonconvexity often implies that distributed solution methods might not be provably convergent to KarushKuhn-Tucker points. To address (c1), and to facilitate the development of provably stable and optimal distributed solution methods for AEGs, a first step is to develop convex relaxations and linear approximations of pertinent nonconvex problems [17], [18]. Section 2.1 explains how to appropriately modify existing relaxation/approximation methods to ensure the feasibility of the produced solutions. The next steps propose developing real-time optimization algorithms that resolve the challenges in $(\mathrm{c} 1)-(\mathrm{c} 3)$; these are described in the ensuing subsections.

\subsection{Feedback-Based Online Optimization}

To capture time-varying operation and economic objectives and constraints, the time-varying optimization formalism [9], [10] is leveraged. Specifically, pertinent time-varying convex optimization problems can model well-defined objectives and constraints of DERs located within each cell as well as consistency constraints for electrical quantities that pertain to the cell-to-cell connections.

Capitalizing on this powerful mathematical model, first-order (gradient-descent based) methods can be used to design online algorithms to track optimal solution trajectories of the formulated time-varying problems; however, existing online optimization methods implicitly operate in an open-loop configuration and might still suffer the challenges noted above in (c2)-(c3). A powerful way to address (c2)-(c3) is to suitably modify the first-order methods to accommodate appropriate measurements from the energy grids and DERs [10], [11], [14], [16]. The resultant feedback-based online optimization methods can cope with inaccuracies in the representation of the AC power flow and avoid pervasive metering to gather the state of noncontrollable resources. These optimization algorithms also naturally afford a distributed implementation by leveraging the decomposition of the Lagrangian function associated with the optimization problems. Particularly, the decomposition of the Lagrangian function can lead to a distributed message passing that entails:

(i) Communications between a cell-level control platform and individual customers, which are necessary to optimize customer-level objectives while respecting electrical limits within a cell; and

(ii) Message passing among cells to optimize the flow of power based on economic and reliability targets.

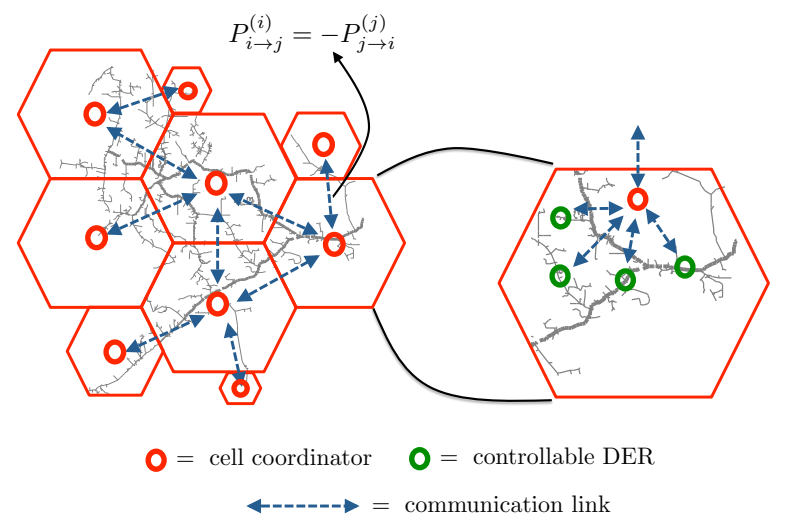

Figure 3. Communications architecture for distributed and real-time optimization of AEGs 
Figure 3 illustrates this architecture, wherein communications among cells occurs because of consistency constraints for electrical quantities that pertain to the cell-to-cell connections-for example, adjacent cells agree on the real and reactive power exchanges at the points of interconnection. Intracellular communication is used to ensure that the set points of the DERs are computed to maximize the given operational objectives while ensuring that electrical limits are satisfied. See, e.g., [10] for intracellular communication and [19] for an example of cell-to-cell consensus-based message passing. As shown in, e.g., [10], optimality and stability results for these online algorithms can be established to track the solution of the formulated time-varying optimization problems.

The idea of accommodating measurements into optimization methods goes back to, e.g., [7], [8]; and it was applied to the real-time optimization of power systems in [11], wherein a centralized controller was developed based on measurement-based projectedgradient methods. Online algorithms were developed in [10], [12], [13], and [14] to find solutions to AC OPF problems; the authors of [10] established results to track solutions of a time-varying linearized AC OPF, and the authors of [14] tracked solutions of a timevarying relaxed AC OPF. Recently, a projectedgradient method on the power flow manifold was proposed in [15], [16]. Although [11], [14], [15], [16] can be implemented in a centralized controller, [10] affords "star" communications between cell-level controllers and DERs.

\subsection{Enabling Real-Time Optimization of AEGs}

To enable real-time optimization of AEGs, the approaches in [10]-[16] should be broadened to accommodate a communication architecture that includes cell-to-cell and cell-to-customer message passing. This can be obtained by dualizing the consensus constraints in the optimization problem that are used to ensure that adjacent cells agree on the power flows from one cell to another. Overall, the resultant feedback-based online optimization methods need to provably track the solution of the convex optimization problems by modeling well-defined objectives and constraints of each cell as well as the consistency constraints for electrical quantities that pertain to the cell-to-cell connections. It is worth emphasizing that the design of the distributed algorithm as well as the overall communications strategy will depend on the type of actors participating in the real-time optimization process-i.e., end customers, cell controllers, or aggregators that participate in the overall optimization process while retaining controllability of their own assets.
The algorithm operates in an asynchronous way to account for different communications latencies and for devices that can be controlled at different timescales (for example, inverter-interfaced devices are controlled at fast timescales, whereas thermostatically controlled loads are controlled every few minutes). Resilience to communications drops and asynchronous operation should be analytically established through pertinent input-to-state stability and tracking results. To this end, the theory for iterative methods that involve gradients with errors can be leveraged. In fact, it can be shown that a packet loss leads to the computation of primal or dual gradient steps with outdated information [30]. A plug-and-play operation wherein cells can switch from an islanded mode to a larger grid-connected mode should be ensured; this can be modeled as a timevarying constraint in the underlying optimization problem. Similarly, flexible operation, wherein a cell (or a portion of an cell) switches to an autonomous control setting during a prolonged communications outage, should be enabled.

Last, distributed optimization will cross-fertilize with the design of (albeit futuristic) market mechanisms to systematically account for payment/rewards to exchange energy exchange and provision ancillary services among autonomous cells. This is in the spirit of transactive energy frameworks [25], which will need to be considerably broadened to accommodate the proposed architecture and to enable a rigorous mathematical analysis of system stability and optimality. For example, the stability analysis in [31] will be extended to account for cell-to-cell interactions.

\section{Control Theory to Ensure Cell Stability and Synchronization}

AEGs pose significant challenges in terms of optimal operation and the analysis of their stability. This is particularly the case when distributed or decentralized control algorithms are used to operate the system (as discussed in Section 2) because these algorithms are inherently asynchronous as a result of communications delays, losses, and distributed (asynchronous) control actions. The typical approach to stability analysis involves analyzing a continuoustime system of differential equations; however, for networked systems with digital controllers, this standard analysis naturally disregards computational and communications latencies as well as asynchronous actions. Another challenge arises because cells must operate autonomously when they are isolated and cannot rely on the frequency and voltage signals from the larger grid. 
To overcome these difficulties, a multi-timescale stability analysis is used. We illustrate the idea using the following multi-timescale control architecture:

- On a slow timescale (e.g., every 5-15 minutes), optimal droop coefficients are computed by a central entity in the cell (using, e.g., the methodology of [20]).

- $\quad$ On a faster timescale (e.g., every second or tens of seconds), optimal power set points are computed using the methods discussed in Section 2.

- On a fast timescale (e.g., every 50-100 msec), the power set points of the controllable assets in the cell are adjusted using the proportional droop controller.

- In real time, the inverters track the given power set point as closely as possible, and they ensure phase and voltage synchronization in case of islanded operation.

The idea is that the stability of the system needs to be ensured by design. To this end, the following considerations need to be made:

(i) The computation of the optimal droop coefficients on the slow timescale must ensure stability of the resulting dynamic system on the fast timescale. Because the time step of the fast-timescale operation is more than 20 milliseconds, a quasi-static approximation can be used-in between the set point update, it is assumed that the system is in its steady state and phasor-based power-flow analysis is applicable. This yields a discrete-time dynamic system that can be analyzed using, e.g., standard boundedinput/bounded-state notions [20].

(ii) To design and analyze real-time controllers, a continuous-time methodology can be used to ensure the stability and synchronization of the cell. The current state of the art provides several alternatives in this context, such as [21], [22], and [23]. The control framework in [23] is a promising approach to enable low-inertia AEGs to operate in a plug-and-play mode while stabilizing cells around set points produced by the real-time optimization algorithms described in Section 2.

Note that these timescales are typically analyzed separately in the literature. This results in suboptimal strategies that do not use the interrelationships between the timescales; hence, a new multi-timescale control framework is needed to design optimal and stable controllers for cellular energy grids.

\section{Big Data Analytics for Energy Systems}

Increasing amounts of heterogeneous sensor data and information are becoming available in energy grids from sources such as smart meters, distributed generation, and smart home energy management systems. Being able to collect, curate, and create actionable information with these data will be critical to creating AEGs. AEGs will need to be able to digest data, evaluate data, and make decisions faster than in real time in both centralized and distributed settings.

The big data analytics that are needed for the envisioned AEG involve three critical steps: i) spatial and temporal characterizing; ii) state estimation and forecasting; iii) autonomous decision-making.

Effectively characterizing the system operation status in both the spatial and temporal domains using big data analytics [26] is the critical first step toward knowledge discovery. Big data analytics in this sense includes advanced concepts such as artificial intelligence and machine learning techniques to help understand the data and make critical decisions in real time. Although the high-volume and high-velocity nature of big data means that many dimensions of information can be extracted, it also brings challenge to processing these data because of possible overfitting. Therefore, in the spatial domain, clustering the system and using optimal sensor selection is critical. The ultimate goal of is to be able to fully observe the system under any conditions using interrelated information to enhance the accuracy and speed of knowledge discovery.

It is understandable that in a networked energy system, there are close correlations among cells. Only a limited number of sensors within each tightly correlated cell are needed to fully monitor the entire cell. As an example, secondary voltage control (SVC) [27] is one way to group such information in each cell. If a power system is represented by a linearized model of the power flow equations, the voltage coupling of the two buses can be used to define the relative electric distance between any two buses. If the reactive power variance at two buses causes coupled voltage variance at those buses, the relative electric distance given this definition is small. Therefore, the buses that have small relative electric distances can be clustered in one SVC cell using a method such as the $\kappa$-means clustering algorithm. Within each SVC cell, the minimum number of sensors can be determined for full observability in state estimation and forecasting. Figure 4 shows an example of SVC clustering and optimal sensor selection. The IEEE 39-bus system is clustered into 7 areas (divided by blue lines), and each area has a limited numbers of sensors (red dots) to ensure full observability. 
In the temporal domain, although many variables can be useful to extract the features of any given system, the time-frequency information is most critical for many energy system applications. Methods such as matching pursuit decomposition (MPD) [28] are very effective for signal time-frequency analysis. Implemented with the Gaussian atom dictionary [29], it can be used to characterize big data represented by the amplitude, time shift, frequency shift, and variance of Gaussian atoms. The basic premises of using MPD for big data analytics in energy systems are as follows:

(i) Any signal can be represented using decomposition with finite iterations and a limited residual.

(ii) The group of parameters that are identified by MPD for any signal can be used for correlation analysis and can therefore overcome data overfitting.

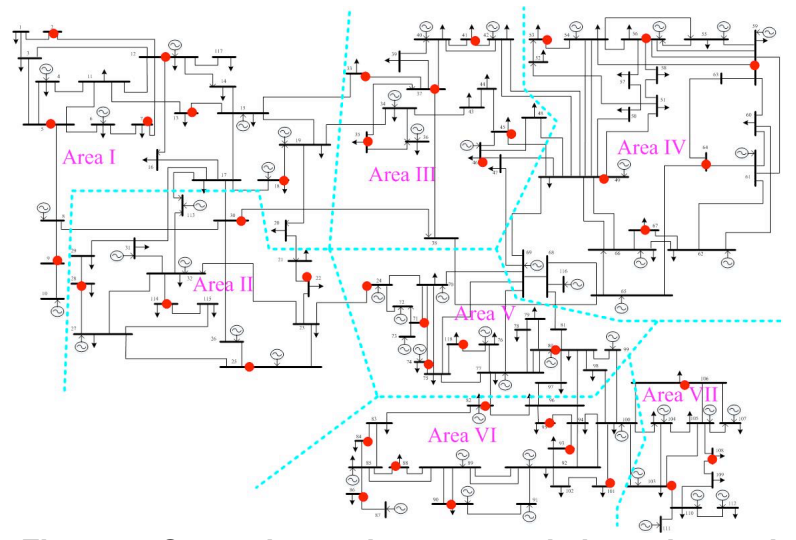

Figure 4. Secondary voltage control clustering and optimal sensor selection using IEEE 39-bus system

Being able to estimate and forecast the system's true states under different aspects of grid operation (including steady states, dynamic and transient conditions) in an online setting (real time) are also essential elements toward automatic decision-making.

To handle nonlinear model analysis, a variety of machine learning-based approaches are used to analyze and forecast system information (e.g., electrical price, load, voltage deviation, congestion). When forecasting a relatively small number of signals, standard statistical methods and human-driven variable selection are sufficient for many applications (for example, relatively simple forecasting models can predict upcoming electrical demand at the level of an entire region); however, if a large amount of data from heterogeneous sources is considered, then even determining the proper correlations and features to use becomes a challenging problem. Also, the scalability and adoptability of a single location's forecast can achieve good results in certain scenarios or a certain time period, but it is not conveniently applied or generalized to other scenarios or different time periods. Therefore, new techniques in machine learningincluding deep neural networks, multikernel learning, Monte Carlo tree searches, dimensionality reduction/data reconstruction, matrix completion, and reinforcement learning - are needed to help estimate and forecast system variables that can decide true operation states.

Innovations in probabilistic decision networks and conditional data analytic structures for energy grids are the keys to enabling autonomous decision-making. Relevant machine learning and signal processing problems for AEGs should be implemented by using distributed algorithms. Similar to Section 2, once the problem is modeled via a well-defined optimization problem, primal-dual-gradient-type methods can be used to design distributed and online algorithms to track optimal solution trajectories of inferential problems and machine learning problems.

\section{Complex Systems Theory and Modeling}

Energy systems include all energy domains (electricity, fuels, thermal) as well as other domains (communications, water, transportation) that influence how energy is generated, distributed, converted, and used. The connections among these domains create interdependencies that challenge overall system design, planning, control, and optimization. Historically, little attention has been paid to the overlap among these areas, but AEGs will need to be able to account for these interdependencies because of the increasing impacts that each energy and infrastructure domain has on another. Similarly, most energy system modeling methods are not conducted on a full time spectrum, wherein changes in transient and dynamic behavior influence steady-state performance. New techniques are needed to address the uncertain and stochastic nature of variable resources and consumer choices across the full time spectrum of operation, from milliseconds to years.

To accurately model the interactions among such disparate energy systems, new modeling approaches are needed that move beyond the single modeling formalism techniques that are most prevalent when representing single energy domains. Multi-paradigm modeling allows for a domain-independent framework that aids in more accurate multisystem representations through multiple formalisms, multiple levels of abstraction, and meta-modeling [24].

The choice of formalism is an important issue in modeling and simulation. Often a certain formalism is used for a particular problem because it seems the most natural or because of historical precedence. For 
example, in power systems the unit commitment and economic dispatch problem is naturally solved through mathematical programming (optimization) techniques; however, mathematical programming would not be a natural fit to solve the dynamic flow of natural gas through a pipeline because this involves solving the partial differential equations of the Navier-Stokes equations. Thus, if the goal is to model the interactions between these two systems, a multi-formalism approach that allows each subsystem to be represented in its most natural form is needed.

Using multiple levels of abstraction is another important concept in modeling and simulating complex systems. This is often performed without prior planning because of data or computational time limitations. For example, a power system planning generation expansion model would not include details of the electromagnetic transients in a generator because (among other reasons) simulating these subsecond phenomena make the simulation of the yearly or decadal time steps of the generation planning decisions computationally infeasible. Therefore, these processes are currently modeled separately. However, there are times, such as planning for contingency events, when considering both of these aspects in an integrated fashion would lead to more optimal decisions or a better understanding of the trade-offs involved in system design and operation.

Therefore, to answer some of the more complex questions about how AEGs would be designed and operated requires the ability to model different levels of abstraction in the same model. Often, this is to be able to answer different questions about the same system under varying scenarios, and thus dynamically changing the level of model abstraction is a significant modeling capability. Figure 5 provides an example of how multi-timescale models of the electricity system could be linked together to make the computation tractable.

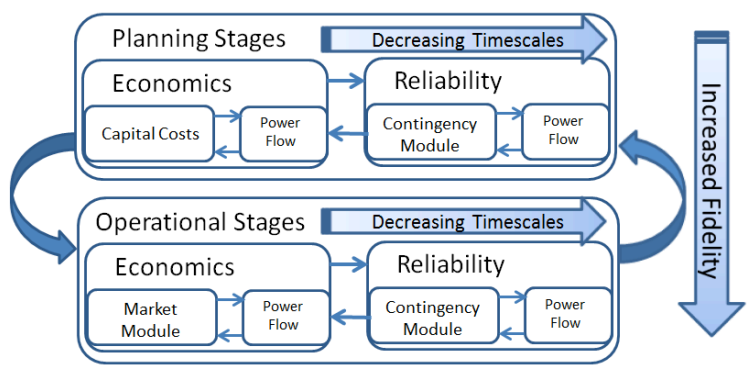

Figure 5. Diagram of how multiple models would interact at different timescales and fidelities

The concept of meta-modeling [32] is very useful for integrating the different levels of model abstraction and modeling formalisms together when considering complex multi-energy systems. Meta-modeling is the process of defining the rules and constructs necessary for creating models. This allows for creating tools that can automatically convert models from one formalism to another or automatically create a model at another level of abstraction. This allows using the same basic simulation data at various levels of aggregation and in multiple simulation formalisms, depending on the current required level of modeling fidelity and the questions under study. Meta-modeling is critical for integrating the different types of models that are necessary to simulate AEGs. The design and operation of AEGs will require further advancements in modeling complex systems because the systems are too large, complex, and costly to create physical representations. Because much of the design will occur through computational simulations, further advancements in modeling and simulating these systems will be necessary to ensure that they are safe, economically efficient, reliable, and robust.

When considering coupled energy infrastructures, the coupling factors among energy carriers are oftentimes assumed constant. This is the case for the fuel-to-power and fuel-to-heat conversion efficiencies for combined-heat-and-power units, for example. However, a number of coupling factors are, in fact, nonlinear: examples include the efficiency and power consumed by a variable-speed water pump, which are nonlinear functions of the pump frequency. Further, the operational region of some type of cogeneration units or absorption and compression chillers might be nonconvex. Multisystem modeling might introduce sources of nonconvexity via bilinear or trilinear terms appearing in equality constraints, which might render the optimization tasks outlined in Section 2 hard to solve. It is thus apparent that trade-offs between the complexity of the modeling approach and achieving flexibility must be taken into account in the system design and operational processes.

\section{Integration to Solve the Challenges of AEGs}

To develop AEGs that are scalable, reconfigurable, and self-organizing, four research areas (Figure 6) have been discussed that, when properly integrated, will play a key role in how future energy systems operate. AEGs rely on scalable, self-configuring cellular building blocks that ensure that each "cell" can selfoptimize when isolated from a larger grid as well as partake in the optimal operation of a larger grid when interconnected. 
A fundamental underpinning of this system is the ability to accurately model the cellular building blocks and their interactions with the rest of the systems so that control, optimization, and forecasting methods might be applied in operation. Complex system theory therefore serves as a means for modeling and simulating the different energy domains and their interactions. These models can then be used for realtime optimization and control of the systems and subsystems, using information gained from big data analytics to provide forecasts that serve as parameters in the control and optimization algorithms as well the algorithmic computational awareness to apply regimeswitching approaches.

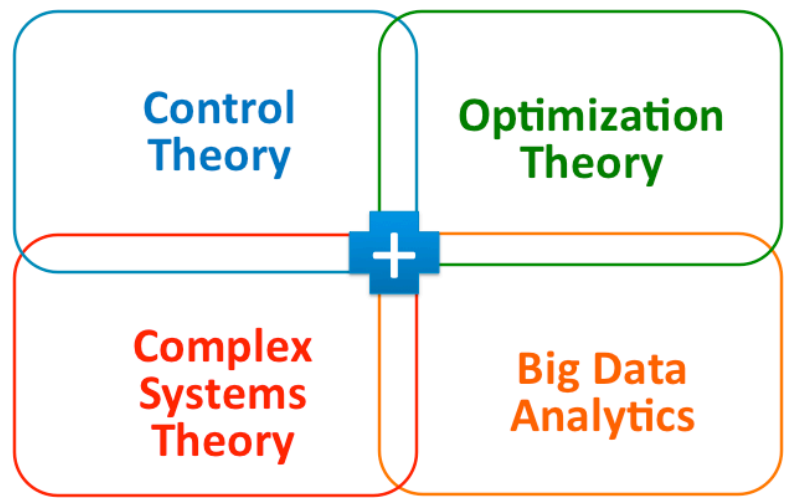

Figure 6. Integration of four technical areas needed to solve the challenges of AEGs

In addition to these four areas of basic research, other important aspects will need further refinement to achieve the full vision of AEGs. New smart grid devices such as the power electronic interfaces of DER inverters will need some embedded autonomous functionality to ensure rapid response to grid anomalies without communications. This would ensure that the devices can respond to voltage and frequency fluctuations quickly enough to damp grid disturbances. To ensure that all these smart devices work together in a reliable and resilient fashion, interoperability and cybersecurity must be embedded into the devices and used across the power system. Finally, because the power system operates using both reliability and economics as mechanisms to ensure stable supply of power at equitable costs, the ownership of devices used in future grid operations and how compensation will flow to devices that provide a range of grid services will need to be further defined. Addressing all of these concerns will be a multiyear effort, but it could provide a power system that will operate in an autonomous fashion that can self-optimize for both reliability and economics while improving resilience, security, and the ability to integrate energy in all forms.

\section{Acknowledgements}

This work was supported by the U.S. Department of Energy under Contract No. DE-AC36-08GO28308 with Alliance for Sustainable Energy, LLC, the Manager and Operator of the National Renewable Energy Laboratory. The U.S. Government retains and the publisher, by accepting the article for publication, acknowledges that the U.S. Government retains a nonexclusive, paid-up, irrevocable, worldwide license to publish or reproduce the published form of this work, or allow others to do so, for U.S. Government purposes.

\section{References}

[1] M. O’Malley, B. Kroposki, B. Hannegan, H. Madsen, M. Andersson, W. D'haeseleer, M. McGranaghan, C. Dent, G. Strbac, S. Baskaran, M. Rinker, "Energy Systems Integration: Defining and Describing the Value Proposition", NREL Technical Report, NREL/TP-5D00-66616, June 2016

[2] B. Kroposki, R. Lasseter, T. Ise, S. Morozumi, S. Papatlianassiou, and N. Hatziargyriou, "Making Microgrids work", IEEE Power and Energy magazine, Vol.6, Issue 3, May-June 2008

[3] J. A. Taylor, S. V. Dhople, and D. S. Callaway, "Power systems without fuel," Renewable \& Sustainable Energy Reviews, vol. 57, pp. 1322-1336, May 2016

[4] E. Dall'Anese, P. Mancarella, and A. Monti, "Unlocking Flexibility: Integrated Optimization and Control of Multienergy Systems", IEEE Power and Energy Magazine, Vol. 15, issue 1, Jan-Feb. 2017

[5] J. Cochran, M. Miller, O. Zinaman, M. Milligan, D. Arent, B. Palmintier, M. O'Malley, S. Mueller, E. Lannoye, A. Tuohy, B. Kujala, M. Sommer, H. Holttinen, J. Kiviluoma, S. K. Soonee, "Flexibility in 21st Century Power Systems. 21st Century Power Partnership", NREL/TP-6A20-61721, 2014

[6] S. Paudyaly, C. A. Canizares, and K. Bhattacharya, "Three phase distribution OPF in smart grids: Optimality versus computational burden," in 2nd IEEE PES Intl. Conf. and Exhibition on Innovative Smart Grid Technologies, Manchester, UK, Dec. 2011. 
[7] A. Jokic, M. Lazar, and P. Van den Bosch, "Realtime control of power systems using nodal prices," Intl. J. of Electrical Power \& Energy Systems, vol. 31, no. 9, pp. 522-530, 2009.

[8] J. Wang and N. Elia, "A control perspective for centralized and distributed convex optimization," in Proc. of 50th IEEE Conf. on Decision and Control, Orlando, FL, Dec. 2011.

[9] A. Simonetto and G. Leus, "Distributed asynchronous time varying constrained optimization," in 48th Asilomar Conference on Signals, Systems and Computers, Nov 2014, pp. 2142- 2146.

[10] E. Dall'Anese and A. Simonetto, "Optimal power flow pursuit,” IEEE Trans. on Smart Grid, May 2016.

[11] A. Bernstein, L. Reyes Chamorro, J.-Y. Le Boudec, and M. Paolone, "A composable method for real-time control of active distribution networks with explicit power set points. part I: Framework," Electric Power Systems Research, vol. 125, no. August, pp. 254-264, 2015.

[12] L. Gan and S. H. Low, “An online gradient algorithm for optimal power flow on radial networks," IEEE Journal on Selected Areas in Communication, vol. 34, no. 3, pp. 625-638, March 2016.

[13] E. Dall'Anese, S. V . Dhople, and G. B. Giannakis, "Photovoltaic inverter controller seeking ac optimal power flow solutions," IEEE Transactions on Power Systems, Volume: 31, Issue: 4, July 2016

[14] Y. Tang, K. Dvijotham, and S. Low, "Real-time optimal power flow," IEEE Trans. on Smart Grid, 2017, DOI: 10.1109/TSG.2017.2704922

[15] A. Hauswirth, S. Bolognani, G. Hug, and F. Dorfler, "Projected gradient descent on Riemannian manifolds with applications to online power system optimization," in 54th Annual Allerton Conference on Communication, Control, and Computing, Sept 2016, pp. 225-232.

[16] A. Hauswirth, A. Zanardi, S. Bolognani, G. Hug, and F. Dorfler, "Online optimization in closed loop on the power flow manifold," in 12th IEEE PES PowerTech conference, 2017.

[17] J. Lavaei and S. H. Low, "Zero duality gap in optimal power flow problem," IEEE Transactions on Power Systems, vol. 27, no. 1, pp. 92-107, 2012.
[18] A. Bernstein, C. Wang, E. Dall'Anese, J.-Y. L. Boudec, and C. Zhao, "Load-flow in multiphase distribution networks: Existence, uniqueness, and linear models," arXiv preprint arXiv:1702.03310, 2017.

[19] E. Dall'Anese, S. V. Dhople, B. B. Johnson, and G. B. Giannakis, "Decentralized Optimal Dispatch of Photovoltaic Inverters in Residential Distribution Systems," IEEE Transactions on Energy Conversion, vol. 29, n. 4, pp. 957-967, Dec. 2014.

[20] K. Baker, A. Bernstein, C. Zhao, and E. Dall'Anese, "Network-cognizant design of decentralized Volt/VAR controllers," in The Eighth Conference on Innovative Smart Grid Technologies (ISGT 2017), 2017.

[21] S. D’Arco, J.A. Suul, O. B. Fosso, “A Virtual Synchronous Machine implementation for distributed control of power converters in SmartGrids", in Electric Power Systems Research, 122, pages 180-197, 2015.

[22] B. B. Johnson, S. V. Dhople, J. L. Cale, A. O. Hamadeh and P. T. Krein, "Oscillator-Based Inverter Control for Islanded Three-Phase Microgrids," in IEEE Journal of Photovoltaics, vol. 4, no. 1, pp. 387-395, Jan. 2014.

[23] M. Colombino, D. Gross, and F. Dörfler. "Global phase and voltage synchronization for power inverters: a decentralized consensus-inspired approach." In Proceedings of the 56th IEEE Conference on Decision and Control, March 2017.

[24] P. Mosterman and H. Vangheluwe, "Computer Automated Multi-Paradigm Modeling: An Introduction", SIMULATION, Vol.80, Issue9, September 2004, pp.433-450

[25] "GridWise Transactive Energy Framework", PNNL Technical Report no. PNNL-22946.

[26] H. Jiang, X. Dai, D. Gao, J. Zhang, Y. Zhang, and E. Muljadi, "Spatial-Temporal Synchrophasor Data Characterization and Analytics in Smart Grid Fault Detection, Identification, and Impact Causal Analysis", IEEE Transactions on Smart Grid, Vol. 7, Issue 5, September 2016, pp. 2525-2536.

[27] J. L. Sancha, J. L. Fernandez, A. Cortes, and J. T. Abarca, "Secondary voltage control: Analysis, solutions and simulation results for the Spanish transmission system," IEEE Transactions on Power Systems, vol. 11, no. 2, pp. 630-638, May 1996. 
[28] S. G. Mallat and Z. Zhang, "Matching pursuits with time-frequency dictionaries," IEEE Trans. Signal Process., vol. 41, no. 12, pp. 3397-3415, Dec. 1993.

[29] S. S. Chen, D. L. Donoho, and M. A. Saunders, "Atomic decomposition by basis pursuit," SIAM Journal on Scientific Computing, vol. 20, no. 1, pp. 33-61, 1998.

[30] N. Gatsis and G. B. Giannakis, "Residential load control: Distributed scheduling and convergence with lost AMI messages," IEEE Transactions on Smart Grid, vol. 3, no. 2, pp. 770-786, 2012.

[31] X. Zhou, E. Dall'Anese, L. Chen, and A. Simonetto, "An Incentive-based Online Optimization Framework For Distribution Grids," IEEE transactions on Automatic Control, 2017. [Online] https://arxiv.org/abs/1705.01482

[32] J. de Lara and H. Vangheluwe, "AToM3: A Tool for Multi-Formalism and Meta-Modelling” Lecture Notes in Computer Science, Volume 2306, pp. 174$188,2002$. 